\title{
Ocular disease in rats: a review
}

\author{
David L. Williams \\ Department of Clinical Veterinary Medicine, University of Cambridge, Madingley Road, Cambridge CB3 OES, UK
}

Address communications to:
David Williams
Tel.: +44 (0)1223 232977
Fax: +44 (0)1223 232977
e-mail:
DoctorDLWilliams@aol.com

\begin{abstract}
The rat Rattus norvegicus is widely used in experimental work, both providing a rodent model for human ocular disease and as a species for toxicologic screening. In addition, the rat is more and more widely kept as a pet, being both friendly and intelligent. Diagnosis and treatment of ocular disease is important for the welfare of individual animals and whole colonies, but also to ensure that spontaneous disease is not compromising experimental work. Here, ophthalmic conditions are reviewed in order to provide a survey of ocular disease in the rat both for veterinary ophthalmologists and for laboratory animal veterinarians and research workers.
\end{abstract}

Key Words: ophthalmic diseases, rat

\section{INTRODUCTION}

The rat Rattus norvegicus has, for many years, been used as an experimental species providing an animal model for human eye disease and, more commonly, in generalized toxicity studies. In both of these cases spontaneous eye disease can complicate experimental or toxicologic work. In addition, ocular lesions in rodents and specifically the rat may often be associated with systemic diseases of importance, both for the individual animal and for the experimental colony. Thus, ocular disease in this species has importance both from a scientific, a laboratory management and an animal welfare perspective. In addition, the rat can make a friendly and intelligent pet, and as such more and more people are keeping rats as pets. They may, in this context, be presented to the veterinarian and, of course, to the veterinary ophthalmologist, should an ocular condition be diagnosed. It is thus important that veterinary ophthalmologists are aware of the range of ocular lesions seen in this species and of possible therapeutic options in such cases.

\section{ORBITAL AND ADNEXAL DISEASE}

\section{Chromodacryorrboea}

A good starting point in considering ocular disease in the rat is that of chromodacryorrhoea (Fig. 1). Understanding the unusual phenomenon of red tear production in this less familiar species demands comprehension of basic anatomy and physiology of tear production in the rat, as well as pathologic changes in disease. Seeing disease in the context of normal physiologic processes is a key point in the investigation of any ocular disease in any species, but perhaps this is particularly so regarding disease involving an unusual process in a less familiar species. For chromodacryorrhoea, investigation requires the consideration of a number of factors, both infectious and noninfectious, which contribute to excess porphyrin production in the rat Harderian gland, the underlying problem in chromodacryorhoea.

The rat eye has three tear-producing glands: the intraorbital, the extraorbital and the Harderian, the latter associated with the third eyelid. Studies in basic biology show that the rat, in common with a number of rodent species, normally produces porphyrin-pigmented and lipid-laden tears predominantly from the Harderian gland. ${ }^{1,2}$ This production is controlled by parasympathetic innervation and can be prevented by the parasympatholytic action of atropine. ${ }^{3}$ Thus, factors which increase parasympathetic drive increase the level of Harderian porphyrin production and can cause chromodacryorrhoea, the overflow of red tears. These stain both the periorbital fur and the fur of the front paws, since rats regularly wipe their eyes with their front paws. Diseases such as mycoplasmosis and silalodacryoadenitis, considered further below, are important causes of chromodacryorrhoea. Thus, red periocular staining in a group of laboratory animals, or less commonly in pet rats, should be a warning sign of potentially severe systemic disease. Other predisposing factors include nutritional deficiencies or other physiologic stresses. It might appear somewhat anomalous that stressors, which must surely increase sympathetic innervation, aggravate a parasympathetically mediated condition. A further influencing factor is prolonged light exposure. As will be discussed further below, prolonged exposure to artificial light has several deleterious effects on the rat eye. One of these is Harderian gland pathology with swelling and necrosis. ${ }^{4,5}$ Porphyrin content is reported to be decreased in these circumstances, but photodynamic effects of Harderian gland 


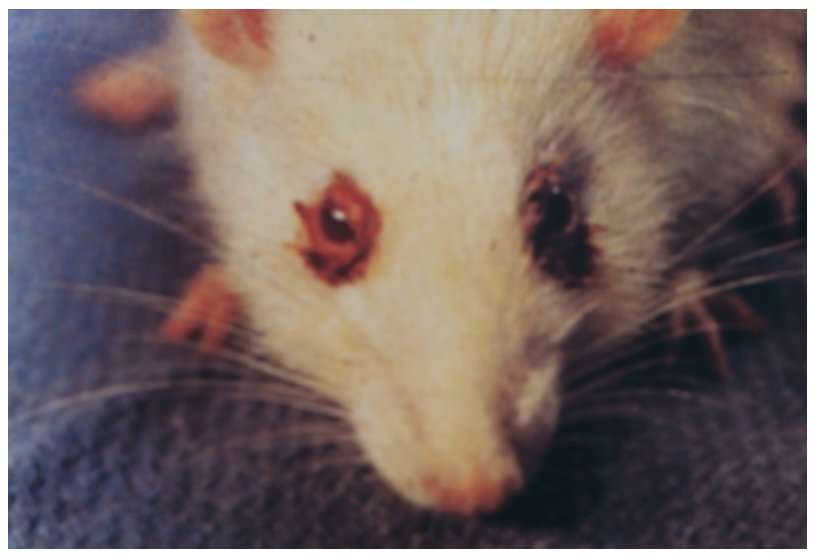

Figure 1. Chromodacryorrhoea (courtesy of Dr L Bauch).

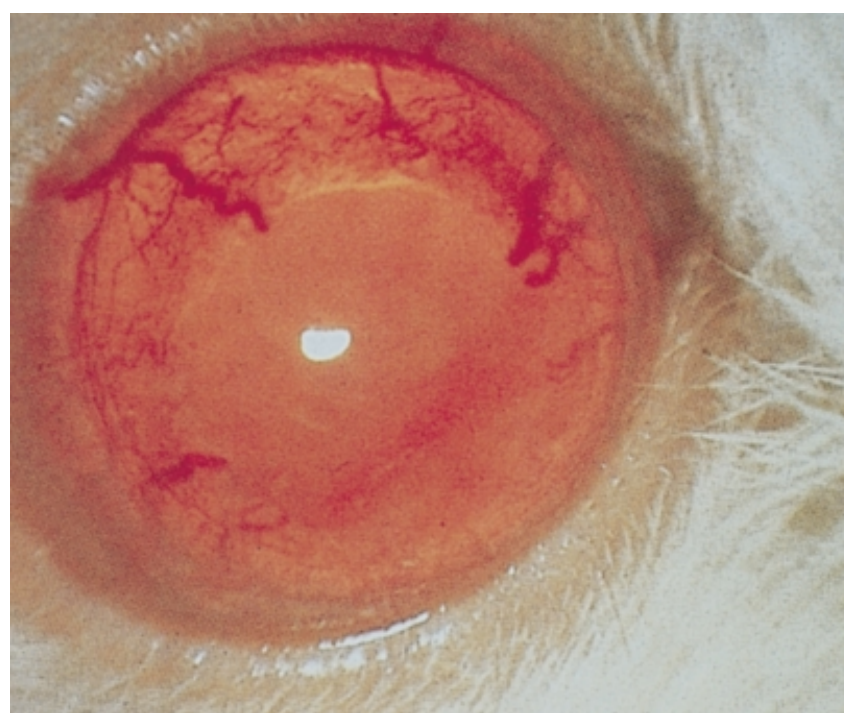

Figure 3. Abnormal iridal vasculature in microphthalmos in the F344 rat.

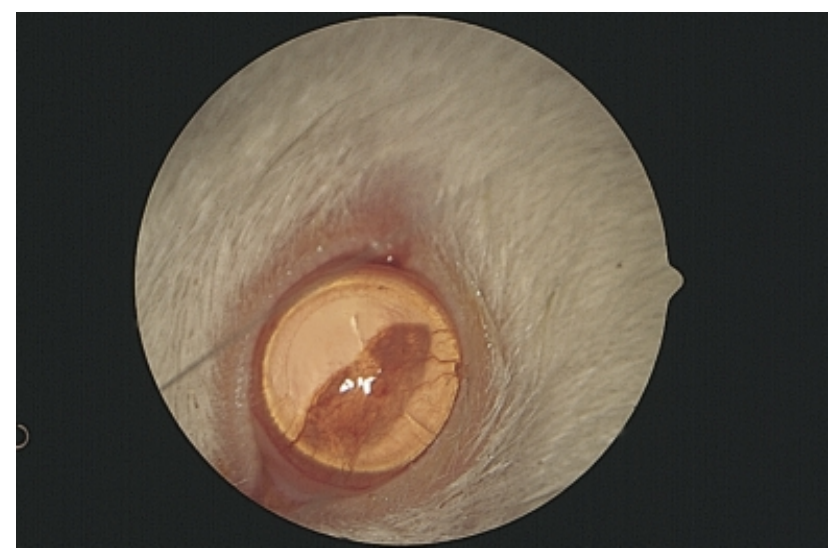

Figure 4. Corneal ulceration as a manifestation of corneal exposure following ketamine/xylazine anesthesia.

porphyrins have damaging effects and photic injury is considered to be a key pathogenic route in ocular surface damage in prolonged light exposure. ${ }^{6}$

Another feature of the rat orbit, important particularly from a laboratory animal perspective, is that of the retrobulbar

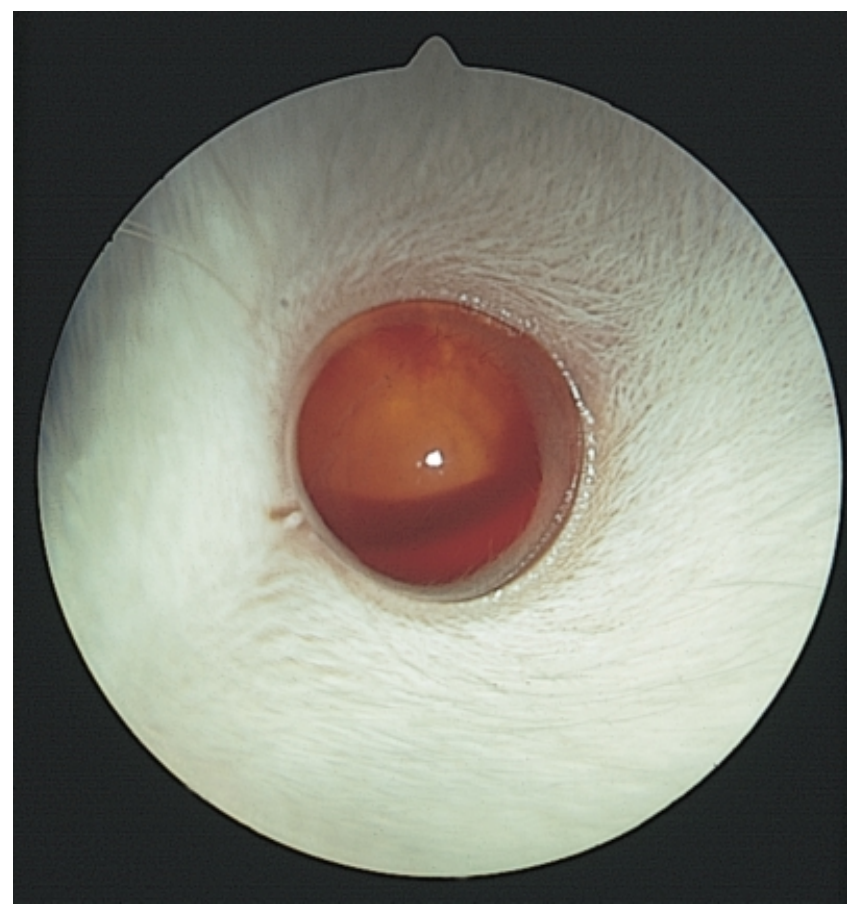

Figure 5. Hyphema in a rat with anterior segment inflammatory disease.

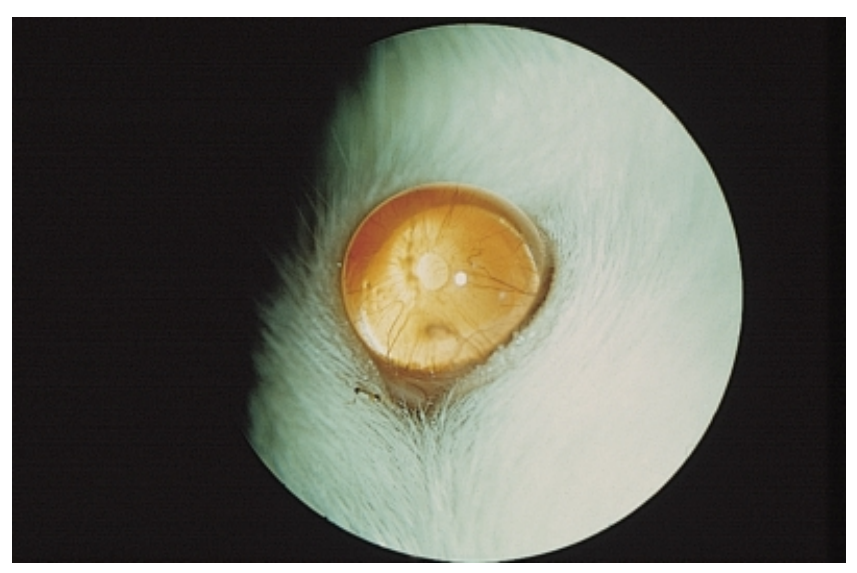

Figure 6. Glaucoma with globe enlargement in a Sprague-Dawley rat.

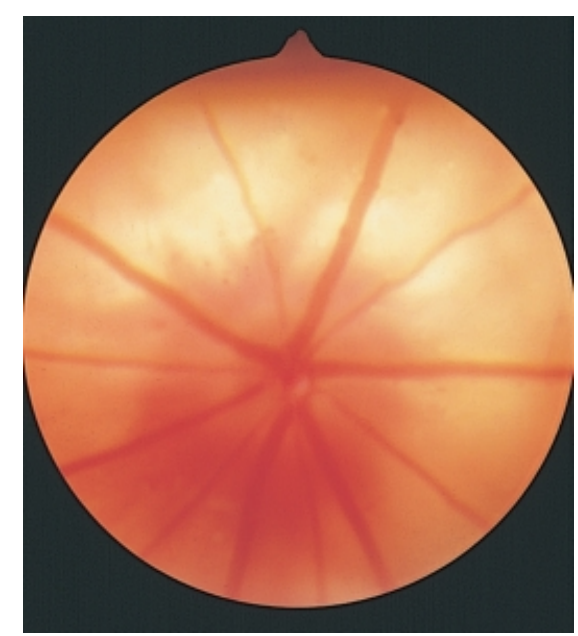

Figure 8. Normal retinal vasculature in an albino rat. 


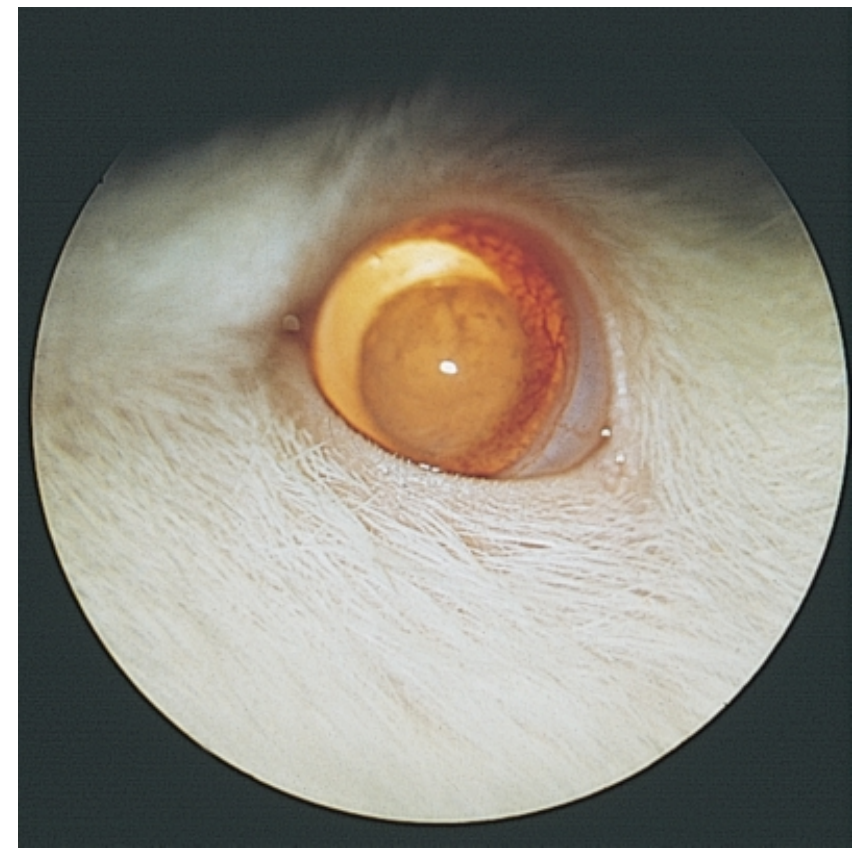

Figure 7. Congenital nuclear cataract in a Sprague-Dawley rat.

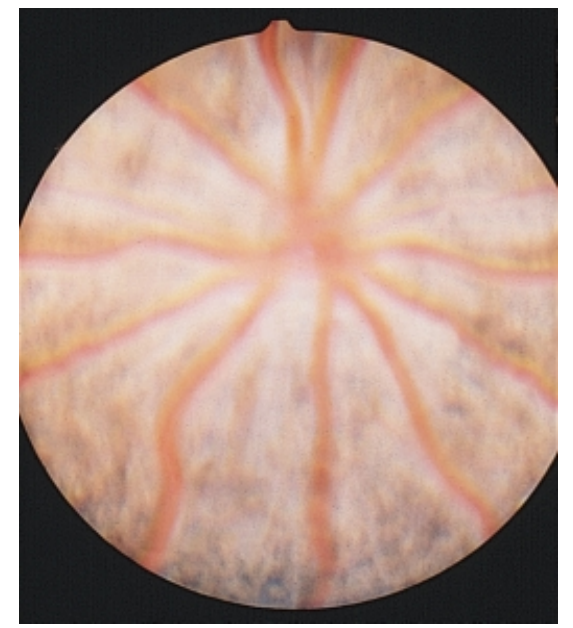

Figure 9. Normal fundus appearance in a pigmented rat strain.

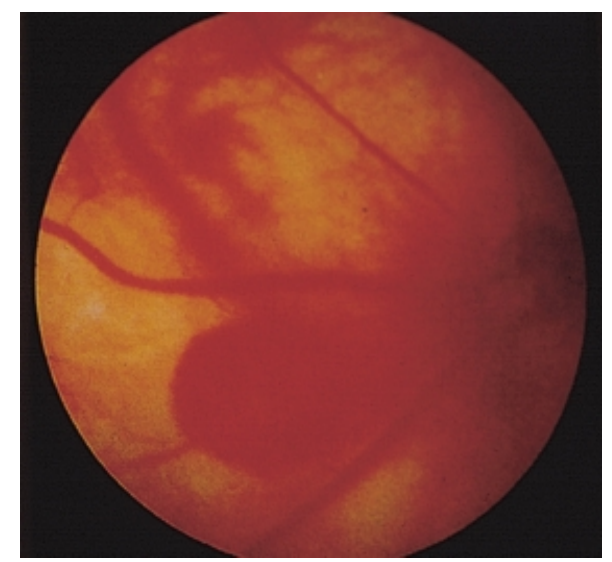

Figure 10. Saccular aneurysms of the retinal vessels in an older rat.

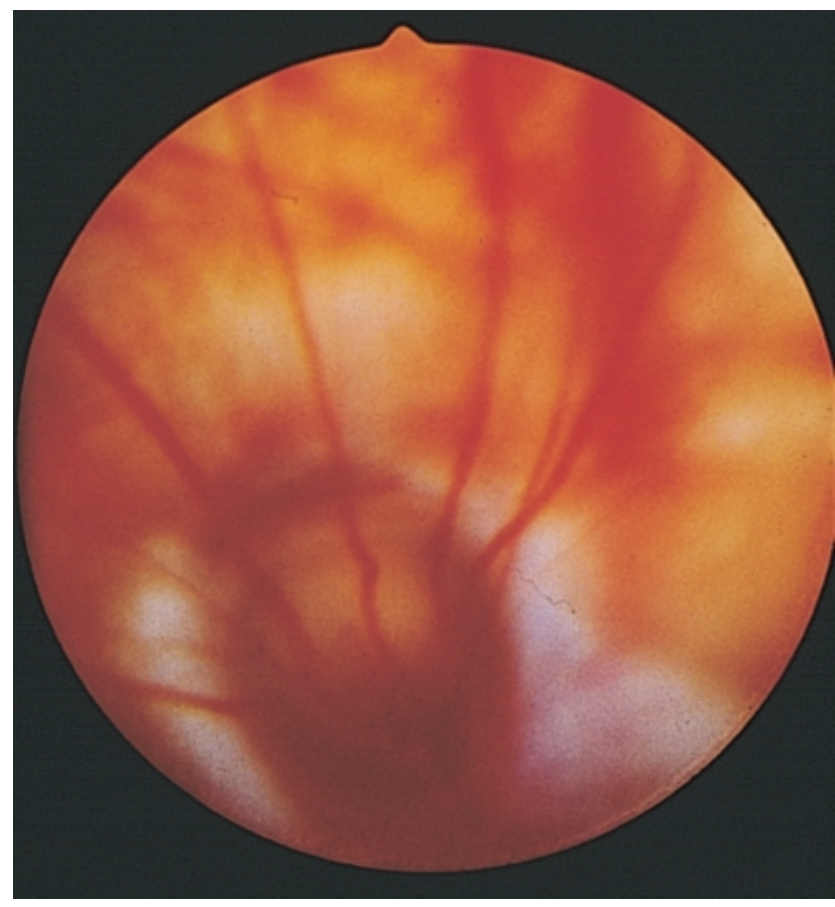

Figure 11. Typical optic nerve coloboma in a Sprague-Dawley rat.

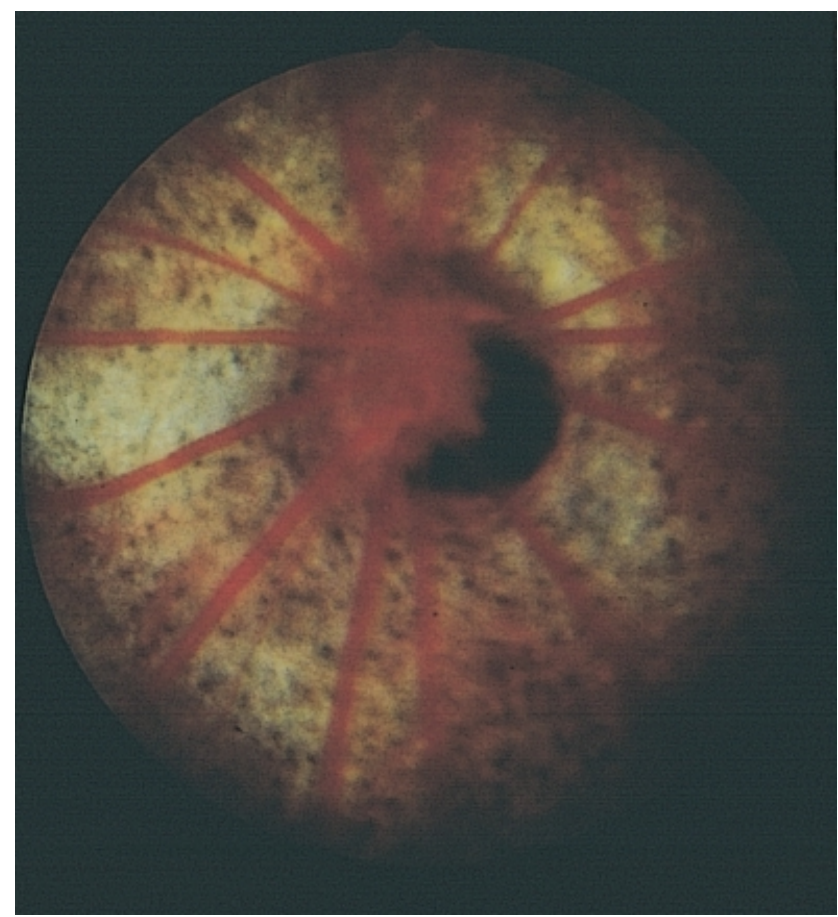

Figure 12. Pigment anomaly at the optic disc within the limits of normal variation in a pigmented strain.

venous sinus, used for blood collection. A 23-g hypodermic needle or capillary tube can be inserted under the upper eyelid at the lateral canthus and directed medially while compressing the jugular vein (Fig. 2). This procedure can be performed many times in experienced hands without apparent detriment. ${ }^{7}$ Other reports, however, have suggested that 


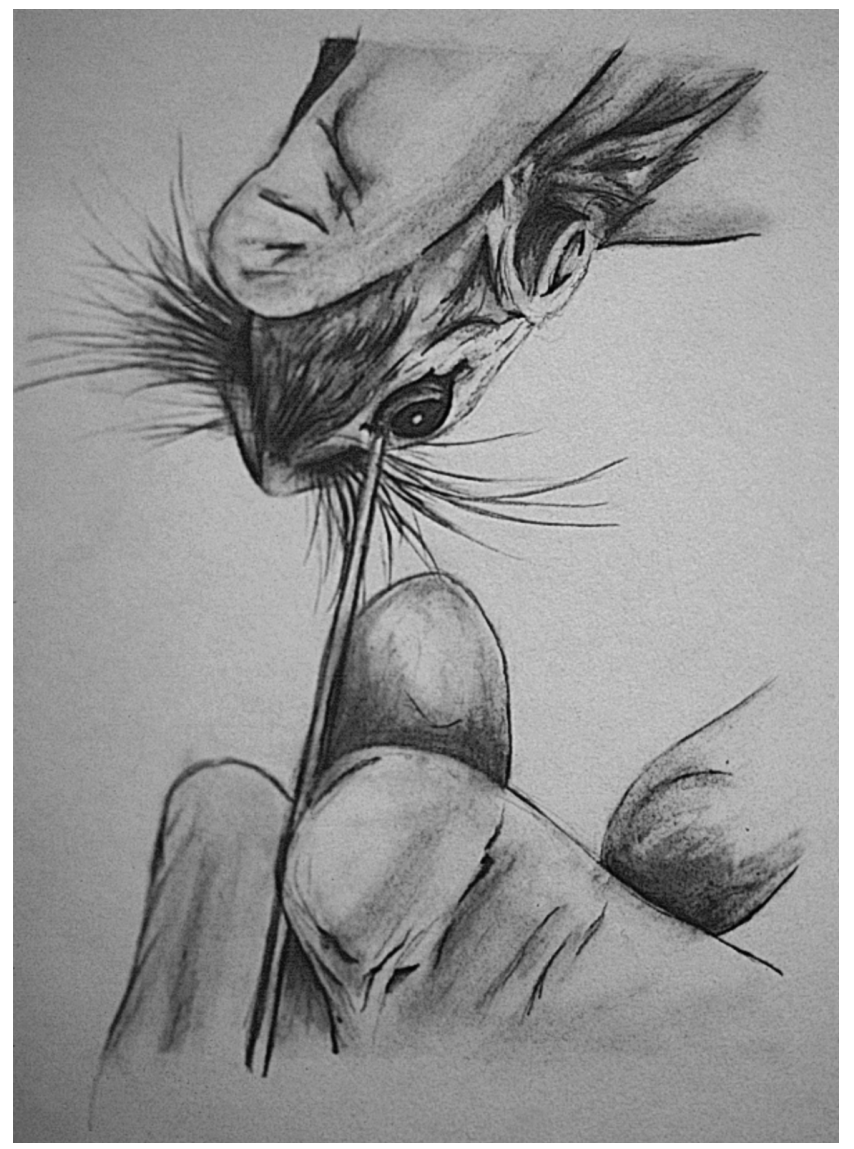

Figure 2. Blood collection from the retrobulbar venous plexus.

postbleeding exophthalmos is seen, ${ }^{8}$ that hemorrhages and subsequent inflammatory foci are found in the puncture track $^{9}$ and that dark focal areas in the nasal fundus subsequent to orbital bleeding may be sequelae of orbital hemorrhage. ${ }^{6}$ This may be related to intraspecies differences: the anatomy of the orbital venous drainage is different in the rat from several other rodents. ${ }^{9}$ While the large sinus in the hamster, gerbil and mouse is easily sampled, the smaller plexus of the rat renders sampling less satisfactory and many consider that the lateral tail vein should be used in preference. In addition to the relatively minor clinical signs after bleeding noted above, more substantial inflammatory disease with subsequent necrosis of the Harderian gland has been reported as associated with orbital bleeding in rats. ${ }^{10}$ This inflammatory disease can be distinguished from that associated with the sialodacryoadenitis (SDA) virus by its localization: foci of inflammation are seen in acini adjacent to the extraocular muscles rather than the generalized nature of inflammation in SDA as discussed further below.

\section{Conjunctivitis}

In rats, as in mice, mycoplasmosis is the most common cause of conjunctivitis not associated with intraocular lesions. ${ }^{11}$ In affected animals a large number of organisms can be isolated from the conjunctival sac, but other bacterial agents are unlikely to have sole pathogenic roles in conjunctival inflammation. ${ }^{12,13}$ Purulent conjunctivitis has been associated with Pasteurella, but this was reported in the context of an underlying mycoplasmal infection. The relative contribution of the two agents to the overall conjunctival pathology is unclear. ${ }^{14}$ Other stressors important in the genesis of respiratoryrelated conjunctivitis include external agencies such as poor ventilation or inexpert handling.

Most important among infectious agents in the rat is the coronaviral infection sialodacryoadenitis. Here conjunctivitis and periorbital swelling are the first signs, followed by sneezing and cervical swelling, this last sign associated with both lymphoadenopathy and salivary gland swelling. This condition is highly contagious and yet is generally selflimiting. These two characteristics mean that the subacute or chronic form is commonly seen throughout colonies rather than an acute form of the disease. The classic epizootic disease, first described in $1961,{ }^{15}$ has high morbidity and low or absent mortality. Nevertheless, it is clearly a major problem in an otherwise pathogen-free colony. Ocular signs may be primary but are often secondary to reduced tear production associated with reactive lacrimal gland hyperplasia. Considerable ductal squamous metaplasia together with periacinar fibrosis occurs during gland repair after inflammation. It is these changes which result in subsequent lacrimal inadequacy. ${ }^{16}$ Resulting keratitis and conjunctivitis lead to chromodacryorrhoea and self-mutilation with attendant further periorbital swelling. The lesions seen in this disease are a manifestation of the reaction between host and infectious agent in the context of the conditions in which the animal is housed. Thus, the disease varies between different strains ${ }^{17}$ and in different environments. ${ }^{18}$ Experimentally immunosuppressed animals had delayed onset of signs with less glandular damage, but also a longer time to resolution of lesions. ${ }^{19}$ While this may seem of purely academic interest, these findings have important clinical implications. The introduction of a naive group of rats to a subclinically affected animal house, with the attendant mild immunosuppression linked with moving and rehousing, may provoke a clinical onset of acute disease in these animals. This can result in prolonged shedding of virus, further compromising the situation in an infected colony. Diagnosis of the disease is normally readily achieved through observation of classic pathognomonic signs, but detection of coronavirus antigen or serologic testing of animals are also possible if confirmation is required..$^{20}$ Widespread serologic testing has shown the agent to be present in $45 \%$ of colonies within the UK, although incidence of overt disease is lower, as might be expected of a chronic subclinical infection. ${ }^{21}$

\section{THE GLOBE}

\section{Microphthalmos and anophthalmos}

Several experimental models of microphthalmos exist in the rat but in addition to these, spontaneous microphthalmos also occurs as an incidental finding in standard nonmicrophthalmic 
strains. One example is the F344 rat in which microphthalmos occurs with a significant frequency. Surprisingly, it is seen in females more than males and in the left eye more frequently than the right; ${ }^{22}$ the embryologic explanation for this remarkable finding is not obvious. Clinical features of mild microphthalmos in these rats include microcornea, engorged episcleral vessels and abnormal vasculature in the iris and posterior segment, as well as reduced globe size (Fig. 3). Spontaneous inherited microphthalmos may thus complicate research programs into other ocular abnormalities or in toxicology studies. From an individual animal perspective, microphthalmic rats often develop defective tear drainage and microbial contamination of their unusually large and deep conjunctival sacs. ${ }^{23,24}$

Anophthalmos has been reported in the rat as in many other species, ${ }^{25}$ but generally in specifically anophthalmic strains rather than occurring as an incidental finding in otherwise normal rats, as does microphthalmos.

\section{Corneal disease}

A substantial proportion of corneal lesions encountered in the rat occur secondary to other ocular or systemic disease. Thus, both conjunctivitis and inflammation of periocular glands often have substantial corneal involvement.

Young Sprague-Dawley, Wistar and Fischer 344 rats have been reported as having subepithelial mineralization in $5-15 \%$ of males and $6-10 \%$ of females. This mineralization, seen between 7 and 26 weeks of age, was reported in some rats to be manifest as fine basophilic granules at the interface between epithelium and stroma, while in others a coarser deposit was noted in either the subepithelial stroma or in the epithelium itself. ${ }^{26-28}$ Another paper compared similar dystrophic corneal mineralization in different strains of mice, finding $26 \%$ of DBA, $16 \%$ of $\mathrm{C} 3 \mathrm{H}$ and $10 \%$ of $\mathrm{Balb} / \mathrm{c}$ mice to have mineralized opacity. ${ }^{29}$ The authors suggested in this report that these lesions were caused by excess ammonia in cage bedding. Another report comparing corneal degeneration in different rat strains in the same environment demonstrated calcium deposition in the subepithelial stroma of the interpalpebral cornea in Wistar and Sprague-Dawley but not Lewis and Long-Evans strains. ${ }^{30}$ While the interpalpebral location of the lesion suggests that environmental factors are important, the interstrain variation suggests that genetic factors in addition to environmental influences are responsible for this corneal lesion. None of these animals showed any clinical abnormalities and thus it might be suggested that this is not of particular importance. From a research perspective, however, they are significant; findings of superior stromal calcium deposition in otherwise normal rodent eyes need to be taken into consideration when evaluating pathologic change in research models.

More severe exposure keratopathy in rodents can manifest as interpalpebral corneal ulceration after prolonged anesthesia (Fig. 4), especially with xylazine and ketamine during which the lids are naturally kept wide open, with a degree of exophthalmos contributing to this lagophthalmos unless the lids are taped shut. Alternatively, an ocular surface protectant lubricant such as the lanolin-based tear replacement ointment lacrilube can be used to prevent ulceration.

Other corneal lesions which have been reported in the rat include epithelial inclusion cysts, dermoids and corneolimbal tumors. ${ }^{26}$ Inclusion cysts have been reported to occur as the result of trauma or as congenital lesions consisting of a keratinized stratified squamous epithelial-lined cyst. ${ }^{31}$ These must be differentiated from neoplastic lesions such as squamous cell papillomas or more invasive carcinomata with dyskeratosis and keratin pearl formation. ${ }^{26} \mathrm{~A}$ dermoid has been reported only once as a haired limbal plaque in one rat. ${ }^{32}$ This paucity of reported cases is surprising given the large number of rats examined in toxicologic studies, work which has given rise to several reports detailing ocular lesions in large series of rats. The lack of reports thus suggests that the incidence is probably truly low compared, for instance, with the guinea pig where a substantial number of cases of dermoid have been reported. ${ }^{33-36}$

\section{Uveal disease}

Anterior uveal disease in the rat involves either congenital anomalies or inflammatory disease. The former include keratolenticular adhesions, as discussed above with regard to microphthalmos or persistence of the pupillary vasculature. Intraocular inflammatory disease may originate in the uvea or arise secondary to corneal perforation. Keratoconjunctivitis may cause such intraocular penetration with associated panuveitis if very severe. Staphylococcal or Streptococcal infections have been implicated in such intraocular spread of disease. ${ }^{37,38}$ Some studies report a high incidence of inflammatory lesions in the anterior segment of rats and suggest it is the most common ophthalmic condition in these animals (Fig. 5).

Blood in the anterior segment should not necessarily be taken to indicate uveal inflammation as it is often associated with persistent embryonic vasculature around the lens and iris. Similarly, blood in the vitreous is often related to persistent hyaloid vessels.

Uveal melanomas have been reported in the Fischer 344 rat, arising from either the iris or from the ciliary body. These tumors have been seen in asbestos chrysolite-treated and ethyl acrylate-treated toxicology study rats, but also significantly in control animals. Such a study shows the importance of viewing toxicologic findings in the context of spontaneous disease. It may be that these lesions are a spontaneous finding and not a toxic change. Spontaneous intraocular melanomas have been reported in both eyes of one Sprague-Dawley rat, ${ }^{39}$ although in this report no confirmatory histopathologic evidence was presented. Schwannomas involving the iris, ciliary body and choroid have been reported in two Sprague-Dawley rats. ${ }^{40}$ In humans the choroid and ciliary body are the prime sites for involvement with this neoplasm and in one of the animals reported here the iris and ciliary body were affected while in the other the choroid was also involved. Here immunohistochemical 
detection of neurofilament and S-100 protein antigens showed the neoplasm to be neural in origin. ${ }^{40}$

\section{Glaucoma}

While noninvasive measurement of intraocular pressure is impossible in the mouse, the rat has an eye which is at, but not beyond, the limits of modern tonometry. ${ }^{41}$ Applanation tonometry using the Tonopen applanation tonometer gave the mean intraocular pressure in Lewis rats as $13.9 \pm 4.2 \mathrm{mmHg}$. This figure may not be accurate for other strains of rat, since a recent study has shown significant differences in intraocular pressure between different strains of mice. ${ }^{41}$ Accurate measurements have yet to be reported for different strains of rat, but until they are some caution should be exercised in extrapolating from one rat strain to another. ${ }^{42}$ Glaucoma has been reported in rats (Fig. 6). ${ }^{43-45}$ However, the failure of aqueous drainage was not primary in most cases, but rather caused by persistent pupillary membranes causing a pupilblock glaucoma, or peripheral anterior synechiae causing an angle-closure glaucoma.

\section{Lenticular disease}

Because of the small diameter of the rodent globe and thus the necessity for a high dioptric power of the lens, the rat lens is extremely large relative to globe size. In the rat, cataracts may occur spontaneously congenitally (Fig. 7$)^{46}$ and in aging animals. ${ }^{47,48}$ These reports concerned the SpragueDawley rat but other strains are affected, from the Sherman $\mathrm{rat}^{49}$ to the $\mathrm{F} 344$ strain. ${ }^{50}$ While at a low incidence, these spontaneous lesions complicate experimental work on cataract induction and toxicologic studies. Lenticular pathology may not be caused by inherent defects of the lens but by other ocular pathology or by environmental factors. In rats with retinal dystrophy or degeneration, cataracts occur secondarily, probably as a consequence of the release of various metabolic biproducts in the posterior segment. ${ }^{51}$

\section{Disease of the posterior segment}

The rat has a holangiotic retina with retinal vessels eminating from the optic disc, seen either against the white sclera in albino animals (Fig. 8) or the pigmented retinal pigment epithelium in strains such as the hooded rat (Fig. 9).

Anomalies of the fetal vasculature of the vitreous and lens are common in the rat. At 5 to 6 weeks of age persistence of the hyaloid vasculature is regularly seen; a single vessel may be present but three to four branches of the hyaloid artery are more commonly seen traversing the vitreous posteriorly from the posterior lens capsule. These vessels regress over the proceeding months but during this period considerable vitreal hemorrhage may occur. Unless very marked, these hemorrhages resolve leaving few remnants. Yellow to brown pigment may, however, remain as hemorrhage is removed by vitreal macrophages. Other posterior segment vascular anomalies include preretinal loops, with $12 \%$ of rats in one study having such an anomaly. ${ }^{52}$

Saccular aneurysms of the retinal vessels have been reported as being a common finding in older laboratory rodents (Fig. 10), and while these might be considered to be congenital, the time point at which they occur is not clear. ${ }^{52}$ Tortuosity of retinal vessels together with preretinal arteriolar loops have also been noted as common retinal abnormalities in CD and Sprague-Dawley rats, ${ }^{53}$ sometimes with associated preretinal hemorrhages.

The other congenital posterior segment defect seen relatively commonly is the coloboma (Fig. 11). Typical (i.e. 6 o'clock) colobomas of the optic disc with or without peripapillary choroidal involvement were found in $0.5 \%$ of Sprague-Dawley rats in one study ${ }^{54}$ while other workers have reported a lower incidence. ${ }^{55}$ Optic disc colobomata may be associated with a cystic microphthalmos,${ }^{56}$ while a small number are associated with iridal colobomas but no other ocular defects. ${ }^{57,58}$

While some retinal lesions seen in young laboratory rodents might be considered congenital, careful examination shows their incidence to rise with increasing age in rat pups. One such lesion is a sharply demarcated pale linear retinal defect seen in young rat pups. Incidence increases from $0.3 \%$ in weanling rats to $3 \%$ at 14 weeks of age. Thus, these should probably be considered as early acquired lesions. Hubert and colleagues describe them histopathologically as focal outer retinal thinning rather than the generalized photoreceptor loss found in the dystrophic animals noted below. ${ }^{59}$ Other workers have, however, described these lesions as retinal dystrophy ${ }^{60}$ or degeneration. ${ }^{61}$ One study has suggested that the focal atrophy represents retinal detachment and reattachment. ${ }^{62}$

Retinal degenerations may be seen in rats either as an inherited lesion or an environmentally induced defect. This dichotomy shows the importance of understanding the background of environmentally induced ocular disease when investigating an inherited defect as a model of human retinal degeneration.

First described by Noell in rats under constant illumination, light-induced retinal degeneration has now been studied extensively in rats and more recently also in mice of different strains. ${ }^{6,64}$ This condition can occur in albino rats exposed to as little as 2 to 3 weeks constant illumination with fluorescent or incandescent lights. Environmental and body temperature is important in defining the rate of degeneration, as is age at onset of photic damage. Even in standard lighting, over one tenth of 2-year-old rats were found to have lesions, although the relationship between light-induced and agerelated retinal degeneration is not particularly well substantiated. Retinal changes such as alterations in vessel caliber, fundic reflectivity and optic disc pallor can be observed ophthalmoscopically after 7 days of continuous light but electroretinography demonstrates changes after only 1 day of this light regime. ${ }^{65}$ Measurement of pupil diameter also shows alterations at an earlier stage than does fundoscopy and is useful, given that it is more readily achieved than electroretinography. The diagnosis of this phototoxic change can be substantiated by noting simple correlates of the 
disease such as increased degeneration in animals in top cages in a stack, or in clear plastic-roofed as opposed to metaltopped cages. Now that the problem is well understood and widely known, the most common cause of photic retinal damage is a malfunction in light timing switches caused by mechanical faults or simply through human error. Even given the understanding of the etiolopathogenesis of this condition it can all too easily complicate and confuse retinal research: the changes seen in the WAG/Rij rat were first thought to be an inherited degeneration ${ }^{66}$ but later discounted as a light-induced retinal change. ${ }^{67}$ Interestingly, even in strains with a defined inherited trait, light has a significant effect. In the RCS rat, discussed further below, environmental light levels have a profound effect on the rate of degenerative change, dark-reared animals having significantly less severe and more protracted degenerative processes than those kept in the light. ${ }^{68}$

Inherited retinal degenerations in rodents have played a considerable role in developing our understanding of retinal function and lesions of retinitis pigmentosa in humans. While the rd and rds mice have perhaps led the field, the rat has had an important part to play also. One of the first conditions fully investigated was retinal degeneration in the Royal College of Surgeons (RCS) rat. ${ }^{69}$ This model is characterized by degeneration of rod outer segments from around 2 weeks postnatally when outer segment debris accumulates between photoreceptors and the RPE. Chimeric RCS/wild type rats showed that the cause of retinal disease lies in the pigment epithelium and not the neurosensory retina: photoreceptors adjacent to normal RPE developed normally while those for which mutant RCS RPE provided phagocytotyic function were seen to degenerate. ${ }^{70}$ Subsequent in vitro studies have confirmed this finding. ${ }^{71}$ Other neuroretinal degenerations in the rat include the photoreceptor degeneration in the Osbourne-Mendel rat, ${ }^{72}$ but these have not been investigated to the same extent as analogous conditions in the rd and rds mouse. They are also not as widespread in the rat population as the mouse mutations, which can often be the cause of blindness in experimental mouse populations. ${ }^{73,74}$

With regard to optic nerve lesions, as with retinal appearance there is a wide normal variation, with changes such as pigment anomalies being within the normal variation in some strains (Fig. 12). Colobomas have already been discussed above but complete optic nerve hypoplasia or aplasia is also seen as a congenital defect, ${ }^{75}$ this possibly occurring secondary to local circulatory abnormalities. ${ }^{76}$ Optic nerve degenerations have also been reported to have a nutritional etiologic component in $\mathrm{F} 344$ rats, ${ }^{77}$ but again the differentiation between a degenerate lesion and a congenital one can be difficult to make. Papilledema has been reported to occur in spontaneously hypertensive rats ${ }^{78}$ and other fundus lesions associated with hypertension have included a red choroidal glow, presumably associated with generalized vessel engorgement (M. Davidson, personal communication).

Optic nerve tumors reported in rats have included meningiomas, ${ }^{79,80}$ these tumors being significantly more malignant than their human counterpart. Again, while the latter report concerns a meningioma following intraorbital nickel bisulfide given in a carcinogenesis study, ${ }^{80}$ the former was a spontaneous finding ${ }^{79}$ showing the importance of placing experimental work in the context of naturally occurring disease.

\section{CONCLUSION}

Spontaneous ocular disease might be thought to be reported relatively uncommonly in the veterinary ophthalmic literature. The large number of animals examined in toxicologic studies, including normal control animals as well as toxicologic subjects, means that relatively rare conditions have been well reported. The importance of diseases such as sialodacryoadenitis and mycoplasmosis also means that ophthalmology has an important part to play in laboratory animal veterinary medicine. Care must be taken when extrapolating from conditions seen in laboratory housed animals to pet rats, but similar principles can be used in investigating ophthalmic disease in a pet animal as in the experimental rat. And, in each, the welfare implications of ocular disease must be carefully considered.

\section{ACKNOWLEDGMENTS}

Dr Lionel Rubin and Mr Peter Lee have most kindly read through and commented on the text of this paper and many of the illustrations used here are courtesy of Mr Lee.

\section{REFERENCES}

1. Sakai T. The mammalian Harderian gland: morphology, biochemistry, function and phylogeny. Archives of Histology of Japan 1981; 44: 299-333.

2. Eida K, Kitutani M. Harderian gland IV. Porphyrin formation from delta-aminolaevulin in the Harderian gland of rats. Chemical Pharmacology Bulletin 1969; 17: 927-931.

3. Harkness JE, Ridgway MD. Chromodacryorrhoea in laboratory rats (Rattus norvegicus): etiologic considerations. Laboratory Animal Science 1980; 30: 841-844.

4. Johnson DD, Rudden PK, O'Steen WK. Photically induced experimental exophthalmos. the role of Harderian and pituitary glands. Investigative Ophthalmology and Visual Science 1979; 18: 1280-1285.

5. O'Steen WK, Kraeer SL, Geller E. Extraocular muscle and Harderian gland degeneration after exposure of rats to continuous fluorescent illumination. Investigative Ophthalmology and Visual Science 1978; 17: 847-856.

6. Kurisu K, Sawamoto O, Watanabe H, Ito A. Sequential changes in the Harderian gland of rats exposed to high intensity light. Laboratory Animal Science 1996; 46: 71-76.

7. Pansky B, Jacobs M, House EL, Tasson JP. The orbital region as a source of blood samples in the golden hamster. Anatomical Record 1961; 139: 409-412.

8. Beynen AC, Baumans V, Haas JWV et al. Assessment of discomfort induced by orbital puncture in rats. In: New Developments in Biosciences: Implications for Laboratory Animal Science. Matinus Nijhoff Publishers, Dordrecht, 1988; 431-436.

9. Van Herck H, Baumans V, Van der Craats NR et al. Histological changes in the orbital region of rats after orbital bleeding. Laboratory Animal 1992; 26: 53-58. 
10. McGee MA, Maronpot RR. Harderian gland dacryoadenitis in rats resulting from orbital bleeding. Laboratory Animal Science 1979; 29: 639-641.

11. Hill A. Experimental and natural infection of the conjunctiva of rats. Laboratory Animal 1974; 8: 305-310.

12. Hunt RD. Dacryoadenitis in the Sprague-Dawley rat. American Fournal of Veterinary Research 1963; 24: 638-641.

13. Young C, Hill A. Conjunctivitis in a colony of rats. Laboratory Animal 1974; 8: 301-304.

14. Roberts A, Gregory BJ. Facultative Pasteurella ophthalmitis in hooded Lister rats. Laboratory Animal 1980; 14: 323-324.

15. Innes JRM, Stanton MF. Acute disease of the submaxillary and Harderian glands (sialodacryoadenitis) of rats with cytomegaly and no inclusion bodies. American fournal of Pathology 1961; 38: 455-468.

16. Jacoby RO, Bhatt PN, Jonas AM. Pathogenesis of sialodacryoadenitis in gnotobiotic rats. Veterinary Pathology 1975; 112: 196-209.

17. Percy DH, Hanna PE, Paturzo F, Bhatt PN. Comparison of strain susceptibility to experimental sialodacryoadenitis in rats. Laboratory Animal Science 1984; 34: 255-260.

18. Jacoby RO, Bhatt PN, Jonas AM. Pathogenesis of sialodacryoadenitis in gnotobiotic rats. Veterinary Pathology 1975; 112: 196-209.

19. Bhatt PN, Jacoby RO. Epizootological observations of natural and experimental infection with sialodacryoadenitis virus in rats. Laboratory Animal Science 1985; 35: 129-134.

20. Hanna PE, Percy DH, Paturzo F, Bhatt PN. Sialodacryoadenitis in the rat: effects of immunosuppression on the course of the disease. American Fournal of Veterinary Research 1984; 45: 2077-2083.

21 Carthew P, Slinger RP. Diagnosis of sialodacryoadenitis virus infection of rats in a virulent enzootic outbreak. Laboratory Animal 1981; 15: 339-342.

22. Gannon J, Carthew P. Prevalence of indigenous viruses in laboratory animal colonies in the United Kingdom 1978-79. Laboratory Animal 1980; 14: 309-311.

23. Lee P. Ophthalmic findings in laboratory animals. Animal Eye Research 1989; 8: 1-12.

24. Sundberg JP, Brown KS, Bates R. Suppurative conjunctivitis and ulcerative blepharitis in 129/J mice. Laboratory Animal Science 1991; 41: 516-518.

25. Rao SR, Sesikeran B. Congenital anophthalmia in CYF rats: a newly identified autosomal recessive mutation. Laboratory Animal Science 1992; 42: 623-625.

26. Yoshitomi K, Boorman GA. The eye and associated glands. In: Pathology of the Fisher Rat. (eds Boorman GA, Eustis SL, Elwell MR et al.) Academic Press, Baltimore, 1990; 112-132.

27. Bellhorn RW, Korte GE, Abrutyn D. Spontaneous corneal degeneration in the rat. Laboratory Animal Science 1988; 38: 46-50.

28. van Winkle TJ, Balk MW. Spontaneous corneal opacities in laboratory mice. Laboratory Animal Science 1986; 36: 248-255.

29. Bellhorn RW, Korte GE, Abrutyn D. Spontaneous corneal degeneration in the rat. Laboratory Animal Science 1988; 38: 46-50.

30. Rubin LF. Ocular abnormalities in rats and mice: a survey of commonly occurring conditions. Animal Eye Research 1986; 5: 15-30.

31. Nichols CW, Yanoff M. Dermoid of a rat cornea. Pathological Veterinaria 1969; 6: 214-216.

32. Alessandarini G. Dermoide centrale della cornea in el cavia cobaya schreb. II Progressia Oftalmologica 1907; 3: 88-89.

33. Chan E. A corneoscleral dermoid in a guinea pig. American fournal of Ophthalmology 1932; 15: 525-526.

34. Gupta BN. Scleral dermoid in a guinea pig. Laboratory Animal Science 1972; 22: 919-921.

35. Otto G, Lipman N, Murphy JC. Corneal dermoid in a hairless guinea pig. Laboratory Animal Science 1991; 41: 171-172.

36. Jones LP. Purulent panophthalmitis in laboratory rats. Fournal of American Veterinary Medical Association 1959; 135: 502-503.
37. Foster H. Purulent keratoconjunctivitis in laboratory rats caused by Micrococcus pyogenes. Fournal of American Veterinary Medical Association 1958; 133: 201-202.

38. Magnusson G, Majeed S, Offer JM. Intraocular melanoma in the rat. Laboratory Animal 1978; 12: 249-252.

39. Chandra M, Frith CH. Histopathologic and immunohistochemical features of two spontaneously generated ocular schwannomas in Spraque-Dawley rats. Laboratory Animal Science 1993; 43: 500-502.

40. Moore CG, Milne ST, Morrison JC. Noninvasive measurement of rat intraocular pressure with the Tono-pen. Investigative Ophthalmology and Visual Science 1993; 34: 363-369.

41. John SWM, Hagaman JR, MacTaggart TE et al. Intraocular pressure in inbred mouse strains. Investigative Ophthalmology and Visual Science 1997; 38: 249-253.

42. Addison WH, How HW. Congenital hypertrophy of the eye in an albino rat. Anatomical Record 1926; 32: 271-274.

43. Young C, Festing MFW, Barnett KC. Buphthalmos (congenital glaucoma) in the rat. Laboratory Animal 1974; 8: 21-31.

44. Hartman HA. Naturally occurring cataracts in the term fetal rat. 7ournal of American Veterinary Medical Association 1968; 153: 832840.

45. Balazs T, Rubin L. A note on the lens in aging Sprague-Dawley rats. Laboratory Animal Science 1971; 21: 267-263.

46. Taradach C, Regnier B. Perraud. Eye lesions in Sprague-Dawley rats: type and incidence in relation to age. Laboratory Animal 1981; 15: $285-287$.

47. Balazs T, Ohtake S, Noble JF. Spontaneous lenticular changes in the rat. Laboratory Animal Care 1970; 20: 215-219.

48. Yosimoto K, Boorman GA. Eye and associated glands. In: Pathology of the Fischer Rat. (eds Boorman GA, Eustis SL, Elwell MR et al.) Academic Press, Baltimore, 1990; 112-132.

49. Zigler JS, Hess HH. Cataracts in the Royal College of Surgeons rat: evidence for initiation by lipid peroxidation products. Experimental Eye Research 1985; 41: 67-76.

50. Bellhorn RW. Ophthalmological disorders of exotic and laboratory animals. Veterinary Clinics of North America 1973; 3: 345-356.

51. Matusi K, Kuno H. Spontaneous fundus abnormalities in rats. Animal Eye Research 1987; 6: 35-41.

52. Hubert M-F, Gillet J-P, Durand-Cavagna G. Spontaneous retinal changes in Sprague-Dawley rats. Laboratory Animal Science 1994; 44: 561-567.

53. Taradach C, Regnier B. Perraud. eye lesions in Sprague-Dawley rats: type and incidence in relation to age. Laboratory Animal 1981; 15: $285-287$.

54. Wyse JPH, Hollenberg MJ. Complicated colobomatous microphthalmos in the BW rat. American fournal of Anatomy 1977; 149: $377-412$.

55. Kuno H, Usui T, Eydelloth RS. Spontaneous ophthalmic lesions in young Sprague-Dawley rats. Fournal of Veterinary Medical Science 1991; 53: 607-614.

56. Rubin LF. Atlas of Veterinary Opbthalmoscopy. Lea \& Febiger, Philadelphia, 1974; 367-397.

57. Hubert M-F, Gillet J-P, Durand-Cavagna G. Spontaneous retinal changes in Sprague-Dawley rats. Laboratory Animal Science 1994; 44: 561-567.

58. Schardein JL, Lucas JA, Fitzgerald JE. Retinal dystrophy in Sprague-Dawley rats. Laboratory Animal Science 1975; 25: 323-326.

59. Lin WL, Essner E. An electron microscopic study of retinal degeneration in Sprague-Dawley rats. Laboratory Animal Science 1987; 37 : 180-186.

60. LaVail MM, Gorrin GM, Repaci MA, Yasumura D. Light-induced retinal degeneration in albino mice and rats: strain and species differences in degenerative retinal disorders. Progress in Clinical Biological Research 1987; 247: 439-454. 
61. Kobayashi K, Ban J, Kuse H, Kawai Y, Hori M. Differences in sensitivity of various ophthalmic methods of detecting light-induced retinal damage in rats. Animal Eye Research 1993; 13: 17-28.

62. Lai Y-L, Jones AM. A rat model for hereditary retinal degeneration. Advances in Experimental Medicine and Biology 1977; 77: 115-136.

63. LaVail MM. Absence of an inherited retinal degeneration in the WAG/Rij rat. Experimental Eye Research 1987; 44: 465.

64. Dowling JE, Sidman RL. Inherited retinal dystrophy in the rat. Fournal of Cell Biology 1962; 14: 73-109.

65. Herron WL, Reigel BW, Myers OE, Rubin ML. Retinal dystrophy in the rat: a pigment epithelial disease. Investigative Ophthalmology and Visual Science 1969; 8: 595.

66. Mullen RJ, LaVail MM. Inherited retinal dystrophy: primary defect in pigment epithelium determined with experimental rat chimeras. Science 1976; 192: 799-801.

67. Edwards RB, Szamier RB. Defective ingestion of isolated rod outer segments by RCS rat RPE in culture. Science 1977; 197: 1001-1003.

68. Von Sailman L, Grimes P. Spontaneous retinal degeneration in mature Osbourne-Mendel rats. Archives of Ophthalmology 1972; 88: 404-409.

69. Schmidt RE. Diagnostic exercise: retinal degeneration in outbred mice. Laboratory Animal Science 1983; 33: 433-434.

70. Shibuya K, Tajima M, Yamate J. Unilateral optic nerve aplasia in two young SIc: Wistar rats. Veterinary Pathology 1989; 26: 518-520.

71. Shibuya K, Tajima M, Yamate J. Unilateral atrophy of the optic nerve associated with retrograde and anterograde degenerations in the visual pathways in SIc: Wistar rats. Fournal of Veterinary Medical Science 1993; 55: 905-912.

72. Lee EW, Render JA, Garner CD et al. Unilateral degeneration of the retina and optic nerve in Fischer-344 rats. Veterinary Pathology 1990; 27: 439-444.

73. Irinodona K. On the fundus: changes of spontaneously hypertensive rats compared with essential hypertension in man. Acta 5th Afro-Asian Congress of Ophthalmology, Tokyo.

74. Greaves P, Faccini JM. Rat Histopathology: a Glossary for Use in Toxicity and Carcinogenicity Studies. Elsevier, Amsterdam.

75. Noell WK, Walker VS, Kang BS, Berman S. Retinal damage by light in rats. Investigative Ophthalmology 1965; 5: 450-473.

76. Yoshitomi K, Everitt JI, Boorman GA. Primary optic nerve meningiomas in F344 rats. Veterinary Pathology 1991; 28: 79-81.

77. Albert DM, Gonder JR, Papale J et al. Induction of ocular neoplasms in Fischer rats by intraocular injection of nickel bisulphide. Investigative Ophthalmology and Visual Science 1982; 22: 768-782.

78. Heywood R. Glaucoma in the rat. British Veterinary fournal of 1975 ; 131: 213-221.

79. Losco PE, Troup CM. Corneal dystrophy in Fischer 344 rats. Laboratory Animal Science 1992; 38: 702-710.

80. Heywood R. Retinal detachment in the rat. Laboratory Animal 1976; 10: 389-392. 